\title{
TENSOR PRODUCTS OF CLEAN RINGS
}

\author{
MASSOUD TOUSI ${ }^{b c}$ and SIAMAK YASSEMI ${ }^{a c *}$ \\ a Department of Mathematics, University of Tehran, Tehran, Iran \\ ${ }^{b}$ Department of Mathematics, Shahid Beheshti University, Tehran, Iran \\ ${ }^{c}$ Institute for Studies in Theoretical Physics and Mathematics, Tehran, Iran
}

(Received 26 January, 2005; accepted 9 June, 2005)

\begin{abstract}
A ring is called clean if every element is the sum of an idempotent and a unit. It is an open question whether the tensor products of two clean algebras over a field is clean. In this note we study the tensor product of clean algebras over a field and we provide some examples to show that the tensor product of two clean algebras over a field need not be clean.
\end{abstract}

2002 Mathematics Subject Classification. 13A99, 13F99.

1. Introduction. Throughout this paper, $R$ is commutative ring and we use $\operatorname{Min}(R)$ to denote the set of minimal prime ideals of $R$. We say $R$ is quasi-local (resp. semi-local) if the set of maximal ideals of $R$ has only one element (resp. finitely many elements). An element in $R$ is called clean if it is the sum of a unit and an idempotent. Following Nicholson, cf. [4], we call the ring $R$ clean if every element in $R$ is clean. Examples of clean rings include all zero-dimensional rings (i.e. every prime ideal is maximal) and local rings. Clean rings have been studied by several authors, for example [4], [2], and [1]. It is an open question whether the tensor product of two clean algebras over a field is clean, cf. [2, Question 3]. The main purpose of this note is to prove Theorem 1, while Theorem 2 and Proposition 3 are used in the proof of Theorem 1. As an application of Theorem 1 we use it to give an example of two clean algebras $A$ and $B$ over a field $F$ where the tensor product $A \otimes_{F} B$ is not clean, see Example 4 . In this paper all algebras are unital.

THEOREM 1. Let $\mathrm{F}$ be an algebraically closed field. Let $\mathrm{A}$ and $\mathrm{B}$ be algebras over $\mathrm{F}$. If $\mathrm{A}$ and $\mathrm{B}$ have a finite number of minimal prime ideals (e.g. $\mathrm{A}$ and $\mathrm{B}$ Noetherian) then the following statements are equivalent:

(i) $\mathrm{A} \otimes_{\mathrm{F}} \mathrm{B}$ is clean.

(ii) The following hold

(a) $\mathrm{A}$ and $\mathrm{B}$ are clean.

(b) A or $\mathrm{B}$ is algebraic over $\mathrm{F}$.

To prove the above Theorem we first recall the following result from [1] and prove Proposition 3.

THEOREM 2. ([1, Theorem 5]) Let $\mathrm{R}$ have a finite number of minimal prime ideals (e.g., $\mathrm{R}$ is Noetherian). Then the following conditions are equivalent.

The research of the first author was supported by a grant from IPM (No. 84130214).

The second author was supported by a grant from IPM (No. 84130216).

*Corresponding author. E-mail: yassemi@ipm.ir 
(i) $\mathrm{R}$ is a finite direct product of quasi-local rings.

(ii) $\mathrm{R}$ is a clean ring.

(iii) $\mathrm{R} / \mathfrak{p}$ is quasi-local for each prime ideal $\mathfrak{p}$ of $\mathrm{R}$.

Proposition 3. Let $\mathrm{A}$ and $\mathrm{B}$ be algebras over a field $\mathrm{F}$. Let $\mathrm{Min}\left(\mathrm{A} \otimes_{\mathrm{F}} \mathrm{B}\right)$ be a finite set and assume that $\mathrm{A} \otimes_{\mathrm{F}} \mathrm{B}$ is clean. Then the following hold.

(i) A or B is algebraic over $\mathrm{F}$.

(ii) $\mathrm{A}$ and $\mathrm{B}$ are clean.

(iii) For any $\mathfrak{m} \in \operatorname{Max}(\mathrm{A})$ and $\mathfrak{n} \in \operatorname{Max}(\mathrm{B})$ the ring $\mathrm{A} / \mathfrak{m} \otimes_{\mathrm{F}} \mathrm{B} / \mathfrak{n}$ is semi-local.

Proof. (i) By Theorem 2 we know that $A \otimes_{F} B$ is semi-local and hence by [3, Theorem 6] $A$ or $B$ is algebraic over $F$.

(ii) Assume that $A$ is algebraic over $F$. Then $\operatorname{dim}(A)=\operatorname{dim}(F)=0$ and so $A$ is clean, cf. [1, Corollary 11]. We know that $\varphi: B \rightarrow\left(A \otimes_{F} B\right)$ is integral. Assume that $\mathfrak{p}_{2} \in \operatorname{Spec}(B)$. Since $\varphi$ is faithfully flat there exists $\mathfrak{q} \in \operatorname{Spec}\left(A \otimes_{F} B\right)$ such that $\mathfrak{q} \cap B=\mathfrak{p}_{2}$. Since $\tilde{\varphi}: B / \mathfrak{p}_{2} \rightarrow\left(A \otimes_{F} B\right) / \mathfrak{q}$ is integral and $\left(A \otimes_{F} B\right) / \mathfrak{q}$ is quasi-local, $B / \mathfrak{p}_{2}$ is quasi-local. On the other hand, since $\varphi$ is faithfully flat and $\operatorname{Min}\left(A \otimes_{F} B\right)$ is finite, $\operatorname{Min}(B)$ is finite too. Therefore, by Theorem $2, B$ is clean.

(iii) By Theorem 2, $A \otimes_{F} B$ is semi-local and so $A / \mathfrak{m} \otimes_{F} B / \mathfrak{n} \cong\left(A \otimes_{F} B\right) /\left(\mathfrak{m} \otimes_{F}\right.$ $\left.B+A \otimes_{F} \mathfrak{n}\right)$ is semi-local.

Proof of Theorem 1. (i) $\Longrightarrow$ (ii) First we show that $A \otimes_{F} B$ has a finite number of minimal prime ideals. Assume $\mathfrak{q} \in \operatorname{Min}\left(A \otimes_{F} B\right)$ and set $\mathfrak{q} \cap A=\mathfrak{p}_{1}$ and $\mathfrak{q} \cap B=\mathfrak{p}_{2}$. Since $A \rightarrow A \otimes_{F} B$ is a faithfully flat homomorphism we have that $\mathfrak{p}_{1} \in \operatorname{Min}(A)$ and for the same reason $\mathfrak{p}_{2} \in \operatorname{Min}(B)$. In addition, $\mathfrak{q} \in \operatorname{Min}\left(\mathfrak{p}_{1} \otimes_{F} B+A \otimes_{F} \mathfrak{p}_{2}\right)$. Since $F$ is algebraically closed $A \otimes_{F} B /\left(\mathfrak{p}_{1} \otimes_{F} B+A \otimes_{F} \mathfrak{p}_{2}\right) \cong A / \mathfrak{p}_{1} \otimes_{F} B / \mathfrak{p}_{2}$ is an integral domain. Therefore $\mathfrak{q}=\mathfrak{p}_{1} \otimes_{F} B+A \otimes_{F} \mathfrak{p}_{2}$. Now the assertion follows from Proposition 3.

(ii) $\Longrightarrow$ (i). Assume that $\mathfrak{q} \in \operatorname{Spec}\left(A \otimes_{F} B\right)$ and set $\mathfrak{q} \cap A=\mathfrak{p}_{1}$ and $\mathfrak{q} \cap B=\mathfrak{p}_{2}$. Then $\mathfrak{p}_{1} \otimes_{F} B+A \otimes_{F} \mathfrak{p}_{2} \subseteq \mathfrak{q}$. Since $A$ and $B$ are clean and $\operatorname{Min}(A)$ and $\operatorname{Min}(B)$ are finite we have that $A / \mathfrak{p}_{1}$ and $B / \mathfrak{p}_{2}$ are quasi-local. Let $\mathfrak{m} / \mathfrak{p}_{1}$ (resp. $\left.\mathfrak{n} / \mathfrak{p}_{2}\right)$ be the unique maximal ideal of $A / \mathfrak{p}_{1}$ (resp. $B / \mathfrak{p}_{2}$ ). Since one of $A$ or $B$ is algebraic over $F$ we have that one of $A / \mathfrak{p}_{1}$ or $B / \mathfrak{p}_{2}$ is algebraic over $F$. Since one of $A / \mathfrak{m}$ or $B / \mathfrak{n}$ is algebraic over $F$ we have $\operatorname{dim}\left(A / \mathfrak{m} \otimes_{F} B / \mathfrak{n}\right)=0$. On the other hand, $F$ is algebraically closed so $A / \mathfrak{m} \otimes_{F} B / \mathfrak{n}$ is an integral domain. Therefore $A / \mathfrak{m} \otimes_{F} B / \mathfrak{n}$ is a field. Now by [5] the ring $A / \mathfrak{p}_{1} \otimes_{F} B / \mathfrak{p}_{2}$ is quasi-local and hence $A \otimes_{F} B /\left(\mathfrak{p}_{1} \otimes_{F} B+A \otimes_{F} \mathfrak{p}_{2}\right)$ is quasilocal. Now the assertion follows from Theorem 2 .

EXAmple 4. Assume that $F=\mathbb{C}$ and $A=B=\mathbb{C}[|x|]$. Then by [1, Proposition 12] $A$ and $B$ are clean. We claim that $A \otimes_{F} B$ is not clean. Otherwise, since $\mathbb{C}$ is an algebraically closed field and $A(=B)$ is Noetherian, by Theorem 1 , we have that $A$ or $B$ is algebraic over $\mathbb{C}$ and hence $A(=B)$ is equal to $\mathbb{C}$. That is a contradiction.

ACKNOWLEDGMENT. It is a pleasure to acknowledge correspondence with W. K. Nicholson who pointed out that the question of Han-Nicholson, cf. [2, Question 3], had not been answered yet. This served to motivate the work reported here. The authors would like to thank the referee for his/her useful comments. 


\section{REFERENCES}

1. D. D. Anderson and V. P. Camillo, Commutative rings whose elements are a sum of a unit and idempotent, Comm. Algebra 30 (2002), 3327-3336.

2. J. Han and W. K. Nicholson, Extensions of clean rings, Comm. Algebra 29 (2001), $2589-2595$.

3. J. Lawrence, Semilocal group rings and tensor products, Michigan Math. J. 22 (1975), 309-313.

4. W. K. Nicholson, Lifting idempotents and exchange rings, Trans. Amer. Math. Soc. 229 (1977), 269-278.

5. M. E. Sweedler, When is the tensor product of algebras local?, Proc. Amer. Math. Soc. 48 (1975), 8-10. 